\title{
Effect of Repeated Gaboxadol Administration on Night Sleep and Next-Day Performance in Healthy Elderly Subjects
}

\author{
Stefan Mathias', Josef Zihl', Axel Steiger*,3 and Marike Lancel' \\ 'Section of Sleep Pharmacology, Max-Planck-Institute of Psychiatry, Munich, Germany; ${ }^{2}$ Section of Neuropsychology, Max-Planck-Institute of \\ Psychiatry, Munich, Germany; ${ }^{3}$ Department of Psychiatry, Max-Planck-Institute of Psychiatry, Munich, Germany
}

\begin{abstract}
Aging is associated with dramatic reductions in sleep continuity and sleep intensity. Since gaboxadol, a selective $G_{A B} A_{A}$ receptor agonist, has been demonstrated to improve sleep consolidation and promote deep sleep, it may be an effective hypnotic, particularly for elderly patients with insomnia. In the present study, we investigated the effects of subchronic gaboxadol administration on nocturnal sleep and its residual effects during the next days in elderly subjects. This was a randomized, double-blind, placebo-controlled, balanced crossover study in 10 healthy elderly subjects without sleep complaints. The subjects were administered either placebo or 15 mg gaboxadol hydrochloride at bedtime on three consecutive nights. Sleep was recorded during each night from 2300 to $0700 \mathrm{~h}$ and tests assessing attention (target detection, stroop test) and memory function (visual form recognition, immediate word recall, digit span) were applied at 0900, 1400, and $1700 \mathrm{~h}$ during the following days. Compared with placebo, gaboxadol significantly shortened subjective sleep onset latency and increased self-rated sleep intensity and quality. Polysomnographic recordings showed that it significantly decreased the number of awakenings, the amount of intermittent wakefulness, and stage I, and increased slow wave sleep and stage 2 . These effects were stable over the three nights. None of the subjects reported side effects. Next-day cognitive performance was not affected by gaboxadol. Gaboxadol persistently improved subjective and objective sleep quality and was devoid of residual effects. Thus, at the employed dose, it seems an effective hypnotic in elderly subjects.

Neuropsychopharmacology (2005) 30, 833-84I, advance online publication, I5 December 2004; doi: I0. I038/sj.npp. I 30064 I
\end{abstract}

Keywords: gaboxadol; hypnotics; sleep stages; EEG spectral analysis; performance

\section{INTRODUCTION}

Insomnia is very common in the elderly population, affecting more than $20 \%$ of the people aged older than 65 years (reviewed in Walsh and Ustun, 1999). This trend is related to dramatic changes that occur in the quantity and quality of sleep during aging (reviewed in Miles and Dement, 1980; Bliwise, 1993). Polysomnographic investigations consistently showed that with advancing age the number and duration of nocturnal awakenings markedly increase, reflecting a decline in sleep maintenance. Furthermore, aging is associated with a progressive decrease in the amount of deep sleep, as indexed both by reductions in slow wave sleep (stages 3 and 4) and slow wave activity (SWA) in the electroencephalogram (EEG) within non-rapid eye movement (non-REM) sleep. To improve their sleep, many

\footnotetext{
* Correspondence: Professor A Steiger, Max-Planck-Institute of Psychiatry, Kraepelinstrasse 2-10, 80804 Munich, Germany, Tel: + 4989 30622236, Fax: + 4989 30622552, E-mail: steiger@mpipsykl.mpg.de Received 3 June 2004; revised 22 October 2004; accepted 12 November 2004

Online publication: 12 November 2004 at http://www.acnp.org/citations/ NPP I | | 204040258/default.pdf
}

elderly patients use hypnotics (Partinen, 1993; Seppala et al, 1993). However, various observations suggest that the therapeutic efficacy of the prescribed hypnotics is far from optimal, particularly for elderly insomniacs. With regard to the acute effects, benzodiazepines promote sleep rapidly and reliably, but suppress REM sleep and deep sleep, as indicated by decreases in slow wave sleep and SWA (reviewed in Parrino and Terzano, 1996; Lancel, 1999). Moreover, many benzodiazepines have been found to produce residual effects the next morning, including daytime sedation, impaired attention, and memory (Gorenstein and Gentil, 1983; Harrison et al, 1985; Roehrs et al, 1986; Hindmarch and Fairweather, 1994). Elderly people appear more sensitive to the sedative effects of hypnotics than the young (Greenblatt et al, 1977; Greenblatt and Allen, 1978; Monane, 1992). Zopiclone and zolpidem are newer hypnotics that lack the benzodiazepine structure, but still interact with the benzodiazepine receptor. Although they seem to have less severe residual effects (Harrison et al, 1985; Billiard et al, 1987; Broadhurst and Cushnaghan, 1987; Scharf et al, 1991; Hindmarch and Fairweather, 1994; Roth et al, 1995; Hemmeter et al, 2000), their sleep effects are qualitatively very similar to the actions of benzodiazepines (reviewed in Lancel, 1999). 
Gaboxadol (4,5,6,7-tetrahydroisoxazolo(5,4-c)pyridin-3ol; THIP) is a GABA analogue and selective $\mathrm{GABA}_{\mathrm{A}}$ receptor agonist. It has an elimination half-life of $1.5-2 \mathrm{~h}$ and maximal plasma levels are reached within $30 \mathrm{~min}$ after oral administration (Schultz et al, 1981). The sleep changes evoked by this compound differ from those of existing hypnotics. In rats gaboxadol dose-dependently increases non-REM sleep, lengthens the duration of the sleep episodes, and elevates SWA in the EEG within non-REMS (Lancel and Faulhaber, 1996; Lancel, 1997; Lancel and Langebartels, 2000). In humans, comparable observations have been made. In young healthy men, a single oral dose of $20 \mathrm{mg}$ gaboxadol was found to increase sleep efficiency, to promote slow wave sleep and enhance the slow frequency components in the EEG within non-REM sleep (Faulhaber et al, 1997). The same dose of gaboxadol has been demonstrated to exert effects on night sleep disrupted by a preceding late afternoon nap. In this homeostatic deprime model of transient insomnia, gaboxadol shortened subjective sleep latency. Polysomnographic recordings revealed that it increased sleep efficiency, which was related to a reduction in intermittent wakefulness and in the number of awakenings, increased slow wave sleep, and augmented SWA in the EEG within non-REM sleep (Mathias et al, 2001). These consistent findings in rats and young men indicate that gaboxadol is able to improve sleep consolidation and to promote deep sleep, without disrupting REM sleep. Therefore, gaboxadol may be an effective treatment strategy for sleep disturbances that are most common in the elderly. Indeed, it has recently been demonstrated that $15 \mathrm{mg}$ gaboxadol reduces self-estimated sleep latency as well the number of awakenings and intermittent wakefulness and augments SWA in the EEG within non-REM sleep in elderly subjects (Lancel et al, 2001). However, an ideal hypnotic should not only improve sleep, but should also be devoid of serious side effects and should not impair daytime functioning. So far, there are no reports on the influence of gaboxadol given shortly before bedtime on performance during the next day.

The aim of the present experiment was to investigate the effect of $15 \mathrm{mg}$ gaboxadol on nocturnal sleep as well as the residual effects on the day following treatment in 10 healthy elderly volunteers without sleep complaints. As residual effects are generally easier to detect after repeated drug administration, we administered gaboxadol $30 \mathrm{~min}$ before bedtime during three consecutive nights and assessed sleep during all the three nights and attention, memory function, and mood during all consecutive days. This investigatorinitiated study was performed at the Max-Planck-Institute of Psychiatry (Munich, Germany) and was sponsored by $\mathrm{H}$ Lundbeck A/S (Copenhagen, Denmark).

\section{MATERIALS AND METHODS}

\section{Subjects}

The participants were recruited by an advertisement in a local weekly newspaper. Ten healthy volunteers (aged 63-78 years), four females (mean age $68.0 \pm 7.1$ ), and six males (mean age $71.2 \pm 3.4$ ) gave written informed consent. The selection of the subjects was based upon polysomnographic screening and thorough psychiatric, physical, and labora- tory examinations. Reasons for exclusion from the study were subjective sleep disturbances, specific sleep disorders (such as sleep-related breathing disorders or periodic leg movement disorder), medical illness, aberrations in hematologic, liver enzyme, kidney function, virology, and thyrotropin-releasing hormone tests, in the EEG and ECG, a personal or family history of psychiatric disorders, substance abuse, recent stressful life events, shiftwork or a transmeridian flight during the preceding 3 months. The subjects were requested to maintain regular bedtimes and to abstain from alcohol prior to and during the experiments.

\section{Study Design}

The experiment has been approved by the ethics committee of the Bavarian Physicians' Council (Bayerische Landesärztekammer). All subjects participated in two study periods that were separated by at least 1 week. Each study period started with an adaptation night, which was followed by three consecutive treatment nights and days in the sleep laboratory. During all nights, the subjects went to bed at $2300 \mathrm{~h}$ and were allowed to sleep till $0700 \mathrm{~h}$. On the treatment nights, the subjects were administered a gelatine capsule at $2230 \mathrm{~h}$, containing placebo (lactose) in one study period and $15 \mathrm{mg}$ gaboxadol (as in all previous studies from our group, the dose is described as gaboxadol hydrochloride; $15 \mathrm{mg}$ gaboxadol hydrochloride corresponds to $12 \mathrm{mg}$ gaboxadol; H Lundbeck A/S, Copenhagen, Denmark) in the other study period according to a randomized, doubleblind, balanced, crossover design. Sleep was recorded during all treatment nights. On the following mornings, 15-30 min after arising, the subjects completed a questionnaire consisting of open questions and self-ratings on $10-\mathrm{cm}$ visual bipolar analogue scales assessing subjective sleep quality and monitoring whether, and, if so, which physical or psychological side effects they had noticed. On the three treatment days, the subjects stayed in the sleep laboratory. Breakfast, lunch, and dinner were provided at 0730, 1200, and $1800 \mathrm{~h}$, respectively. The subjects were allowed their usual intake of caffeine-containing beverages. On each treatment day, the subjects were tested on a 50-min cognitive performance and mood assessment battery beginning at 0900,1400 , and $1700 \mathrm{~h}$. The battery included selective attention tests (target detection, stroop test), shortterm and working memory tests (visual form recognition, immediate word recall, digit span), and a questionnaire assessing mood state (BSKE16E/LSKE6-S), which were administered in random order. All tests were administered by the same experienced examinator, who was unaware of the treatment. Shortly before the start of the first test session, the tests were explained by the examiner.

\section{Sleep Recording}

Polysomnographic recordings consisted of two EEG channels (C3-A2 and C4-A1), vertical and horizontal electrooculograms (EOG), and the submental electromyogram (EMG). These data were recorded using a digital sleeprecording system (Schwarzer Brainlab 3.30) amplifying the signals at a sampling frequency of $250 \mathrm{~Hz}$ with digital highand low-pass filtering at 0.53 and $70 \mathrm{~Hz}$, respectively, for the EEG channels, 16 and $300 \mathrm{~Hz}$ for the EMGs, and 0.095 and 
$30 \mathrm{~Hz}$ for the EOGs. Sleep stages were visually scored from screen per $30 \mathrm{~s}$ according to the criteria of Rechtschaffen and Kales (1968) by two experienced raters who were unaware of the treatment. For technical reasons, the third placebo night of one subject is missing. The missing values have been replaced by the mean of the first two placebo nights.

Power spectra of the C3-A2 EEG derivation were computed using a fast Fourier transformation with rectangular spectral windows of $2.0 \mathrm{~s}$ duration. Preceding the FFT, a linear interpolation between adjacent raw values (sampled at a frequency of $250 \mathrm{~Hz}$ ) was carried out, followed by a second sampling procedure (by program) at a frequency of $256 \mathrm{~Hz}$, yielding 512 values in the $2.0 \mathrm{~s}$ spectral window. With this algorithm, the frequency resolution of the power spectra is $0.5 \mathrm{~Hz}$. Power was computed up to $20 \mathrm{~Hz}$. For each sleep epoch, 15 spectra were computed with the first spectral window starting at the beginning of the epoch and a shift of $2.0 \mathrm{~s}$ between the consecutive spectral windows. The spectra of $2 \mathrm{~s}$ intervals containing visually identified EEG artifacts were eliminated. The spectra of each epoch were averaged. Average EEG power densities and SWA (1.0$4.0 \mathrm{~Hz}$ ) within non-REM sleep were computed for each 2 -h interval. Owing to large interindividual differences in absolute power, the data were normalized by expressing them as a percentage of the average power in the same frequency band within non-REM sleep during the entire placebo nights.

\section{Performance and Mood Assessment Battery}

There is evidence in the literature that sleep disturbances can affect cognition in elderly people, especially attention and memory (Kunz and Herrmann, 2000; Ohyon and Vecchierini, 2002). In addition, elderly people with a mild cognitive impairment typically have difficulties with attention and memory demands (Tuokko and Frerichs, 2000; Hedden and Gabrieli, 2004). Based on this empirical evidence, we focused on the assessment of attention and memory. In the attention domain, we measured visual selective attention without (target detection) and with an executive component (Stroop test). In the memory domain visual working memory (form recognition), verbal shortterm and working memory (digit span forward and backward) and verbal learning (word list) with immediate recall were assessed.

Selective attention, Target detection: In this computerized test, which has a similar structure as the d2-test (Brickenkamp, 1995), a blue triangle and a number of blue bars are presented on screen. The subject has to decide whether an item is a target (triangle points downwards and there are two bars) or not as quickly as possible. The total test duration is $4 \mathrm{~min}$ and $40 \mathrm{~s}$; performance is defined by the total number of correct responses.

Selective attention, Stroop test: This test is based on the findings that it takes longer to call out the color names of color patches than to read words, and even longer to read printed color names when the print ink is in a color different from the name of the color word. The latter phenomenon is attributed to a response conflict or a failure of response inhibition, respectively; more generally, it can be attributed to a difficulty in warding off distractions
(Lezak, 1995). The time required to read 72 black-printed color words (FWL) and to name the color of 72 colored horizontal bars (FSB) as well as of 72 color words printed in a different color (interference, INT) is measured. From FWL and FSB, expected values of, respectively, FSB and INT can be computed (Bäumler, 1985). Subject's scores are $t$-values of the differences between the real and expected FSB (nomination, T-NOM) and INT (selective attention, T-SEL). Three versions exist of this test and these were randomized across the test sessions.

Visual form recognition: This computerized test is similar to the visual discrimination test of Benton et al (1983) for the assessment of visual working memory. The main difference is that it is not a multiple-choice test, but a sequential one-item recognition test. At $6 \mathrm{~s}$ intervals, 10 simple geometric shapes (for example, a circle, triangle, square, rectangle) are consecutively presented on screen. The subject should press a button, when he/she thinks that a form with the same shape and size has already been shown previously. The order of the 10 figures is randomized and, thus, differed between the test sessions. The subject's score is the number of correctly recognized forms in a total of 40 trials. Immediate word recall: In this task, measuring verbal working memory, 10 words are consecutively shown on screen at $2 \mathrm{~s}$ intervals. Immediately thereafter, the subject is asked to recall the words. The test is based on the word list version in the CERAD, a test battery developed for the detection of minimal memory impairments in older subjects (Mooris et al, 1989). Nine different versions of the test exist and each version was used only once within a study period. The subject's performance is calculated on the basis of the number of correctly recalled items.

Digit span: A sequence of digits is read aloud by the examiner at a rate of $1 / \mathrm{s}$. The subject is required to repeat the entire sequence forward or backward, which is related to short-term memory and working memory, respectively. After two sequences of the same length, the number of digits is increased by one. In the forward test, the number of digits is between three and eight and in the backward test between two and seven. The subject's score is the total number of correctly forward or backward repeated sequences. Nine versions exist of both tests and each version was used once within a study period.

BSKE16E/LSKE6-S: This questionnaire (Befindlichkeitsskalierung/Leistungsskalierung anhand von Kategorien und Eigenschaftswörtern zur Zustandserfassung im Rahmen von neuropsychologischer Diagnostik) consists of a list of 11 adjectives, each with seven response categories (ranging from ' 0 ', indicating 'not at all', to ' 6 ', indicating 'extremely') on which subjects can describe how they feel with respect to their mood and their efficiency at that moment. The scale is a revised version of the 'Befindlichkeitsskalierung anhand von Kategorien und Eigenschaftswörtern' (Janke et al, 1999).

\section{Statistical Analysis}

To approach normality, the visually scored and self-rated sleep parameters as well as the performance parameters were transformed with a log transformation $(y=\log 10(x+$ $1)$ ). Most sleep parameters were analyzed by means of a two-factorial repeated-measures analysis of variance 
(ANOVA; Greenhouse Geisser correction) with treatment (two levels: placebo $v s$ gaboxadol) and treatment night (three levels) as within-subjects factors. The performance parameters and some sleep parameters were analyzed by means of a three-factorial repeated-measures ANOVA with treatment (two levels), treatment day/night (three levels), and session or time (three or four levels) as within-subject factors. Where appropriate, ANOVA was followed by tests with contrasts.

\section{RESULTS}

\section{Subjective Sleep Quality}

Analysis of the subjective sleep parameters yielded a significant treatment effect for perceived sleep onset latency $(F(1,9)=7.8, \quad P<0.05)$, self-rated sleep quality $(\mathrm{F}(1,9)=13.3, P<0.01)$, and sleep intensity $(\mathrm{F}(1,9)=5.6$, $P<0.05)$. Independent of the treatment night, sleep latency was shorter and sleep quality and intensity higher during gaboxadol than during placebo treatment (Table 1). Furthermore, ANOVA found a significant interaction effect between the factors treatment and treatment night for rated fitness upon awakening $(\mathrm{F}(2,18)=6.6, P<0.05)$. Pairwise comparisons between consecutive treatment nights by tests with contrasts showed that fitness upon awakening significantly decreased from placebo night 2 to 3 $(P<0.01)$, whereas it did not change across the gaboxadol nights. Consequently, the subjects felt significantly fitter after the third gaboxadol night than after the third placebo night.

None of the subjects reported negative side effects.

\section{Visually Scored Sleep Parameters}

ANOVAs of the visually scored sleep parameters revealed a significant effect of treatment night for total sleep time $(\mathrm{F}(2,18)=4.5, \quad P<0.05)$, sleep efficiency $(\mathrm{F}(2,18)=4.3$, $P<0.05)$, wakefulness $(\mathrm{F}(2,18)=6.1, P<0.05)$, and wakeful- ness after sleep onset $(\mathrm{F}(2,18)=4.9, P<0.05)$. Irrespective of the treatment, total sleep time and concomitantly sleep efficiency gradually decreased, while wakefulness increased across the treatment nights (Table 2). Furthermore, a significant treatment effect emerged for total sleep time $(\mathrm{F}(1,9)=34.5, \quad P<0.001)$, sleep efficiency $(\mathrm{F}(1,9)=36.7$, $P<0.001)$, stage $1 \quad(\mathrm{~F}(1,9)=11.3, \quad P<0.01), \quad$ stage 2 $(\mathrm{F}(1,9)=6.0, \quad P<0.05)$, slow wave sleep $(\mathrm{F}(1,9)=16.5$, $P<0.01)$, wakefulness $(\mathrm{F}(1,9)=16.1, P<0.01)$, wakefulness after sleep onset $(\mathrm{F}(1,9)=6.9, P<0.05)$, the number of awakenings $(\mathrm{F}(1,9)=6.9, P<0.05)$, and movement time $(\mathrm{F}(1,9)=10.7, P<0.01)$. Compared to placebo, gaboxadol produced an overall increase in total sleep time, of approximately $25 \mathrm{~min}$, which was related to a decrease in wakefulness, particularly in wakefulness after sleep onset, and a reduction in the number of awakenings. The increase in total sleep time resulted in an increase in the sleep efficiency index, on average from $78.2 \pm 6.7$ to $83.3 \pm 6.7$, and was associated with increases in stage 2 and slow wave sleep. Furthermore, stage 1 was generally decreased and movement time slightly increased. Gaboxadol did not produce a significant change in the latency to REM sleep nor in the total time spent in this sleep stage.

To examine when the gaboxadol-induced changes in sleep and wakefulness took place, time spent in wakefulness after sleep onset, stage 2, and slow wave sleep were computed for each 2-h interval. After being log transformed, these data were analyzed with a three-factorial repeated-measures ANOVA with the factors treatment, treatment night, and time (four 2-h intervals) as within-subjects factor. For wakefulness, significant effects of treatment $(\mathrm{F}(1,9)=8.7$, $P<0.05)$ and time $(\mathrm{F}(3,27)=11.6, P<0.001)$ were found. As can be seen in the upper plot of Figure 1, wakefulness increased progressively in the course of the placebo and gaboxadol nights and gaboxadol reduced wakefulness throughout the night. For stage 2, only a significant treatment effect emerged $(F(1,9)=7.6, P<0.05)$, indicating that the gaboxadol-produced increase is not restricted to a specific part of the night. In contrast, for slow wave sleep, a

Table I Self-Rated Sleep Quality

\begin{tabular}{|c|c|c|c|c|c|c|c|c|}
\hline & \multicolumn{4}{|c|}{ Placebo } & \multicolumn{4}{|c|}{ Gaboxadol } \\
\hline & Night I & Night 2 & Night 3 & Average & Night I & Night 2 & Night 3 & Average \\
\hline SOL (min) & $29.0 \pm 20.9$ & $23.0 \pm 14.8$ & $33.5 \pm 29.2$ & $28.5 \pm 14.3$ & $12.2 \pm 4.9$ & $17.0 \pm 8.9$ & $25.0 \pm 19.9$ & $18.1 \pm 8.6 *$ \\
\hline TST (h) & $7.0 \pm 0.6$ & $7.0 \pm 0.4$ & $6.5 \pm 1.0$ & $6.8 \pm 0.5$ & $7.3 \pm 0.5$ & $7.2 \pm 0.5$ & $6.9 \pm 0.6$ & $7.1 \pm 0.5$ \\
\hline \multicolumn{9}{|c|}{ 10-cm visual bipolar analog scales } \\
\hline Sleep quality & $7.6 \pm 1.8$ & $8.1 \pm 1.3$ & $6.4 \pm 2.4$ & $7.3 \pm 1.0$ & $8.9 \pm 0.8$ & $7.6 \pm 2.0$ & $8.3 \pm 1.5$ & $8.3 \pm 0.8 *$ \\
\hline Sleep intensity & $7.3 \pm 2.3$ & $7.8 \pm 1.6$ & $6.5 \pm 2.1$ & $7.2 \pm 1.1$ & $8.0 \pm 1.6$ & $7.4 \pm 1.5$ & $7.7 \pm 1.6$ & $7.7 \pm 1.0 *$ \\
\hline Fitness & $8.3 \pm 1.7$ & $8.3 \pm 1.6$ & $6.3 \pm 2.2$ & $7.6 \pm 1.2$ & $7.1 \pm 2.7$ & $7.1 \pm 2.8$ & $7.9 \pm 1.9^{\#}$ & $7.3 \pm 2.2$ \\
\hline
\end{tabular}

Values are means $\pm \operatorname{SD}(n=10)$. Average, mean of three placebo or gaboxadol nights.

SOL, sleep onset latency; TST, total sleep time; NA, number of awakenings.

*Significant difference between the treatments $\left(P<0.05\right.$, ANOVA effect of treatment) and ${ }^{\#}$ significant difference between night 3 of placebo and gaboxadol $(P<0.05$, tests with contrasts). Statistical procedures were applied to log-transformed data. Sleep quality: bad ( 0 ) vs good ( I 0). Sleep intensity: superficial (0) vs deep ( I 0). Fitness upon awakening: numb (0) vs fresh (10). 
Table 2 Visually Scored Sleep Variables

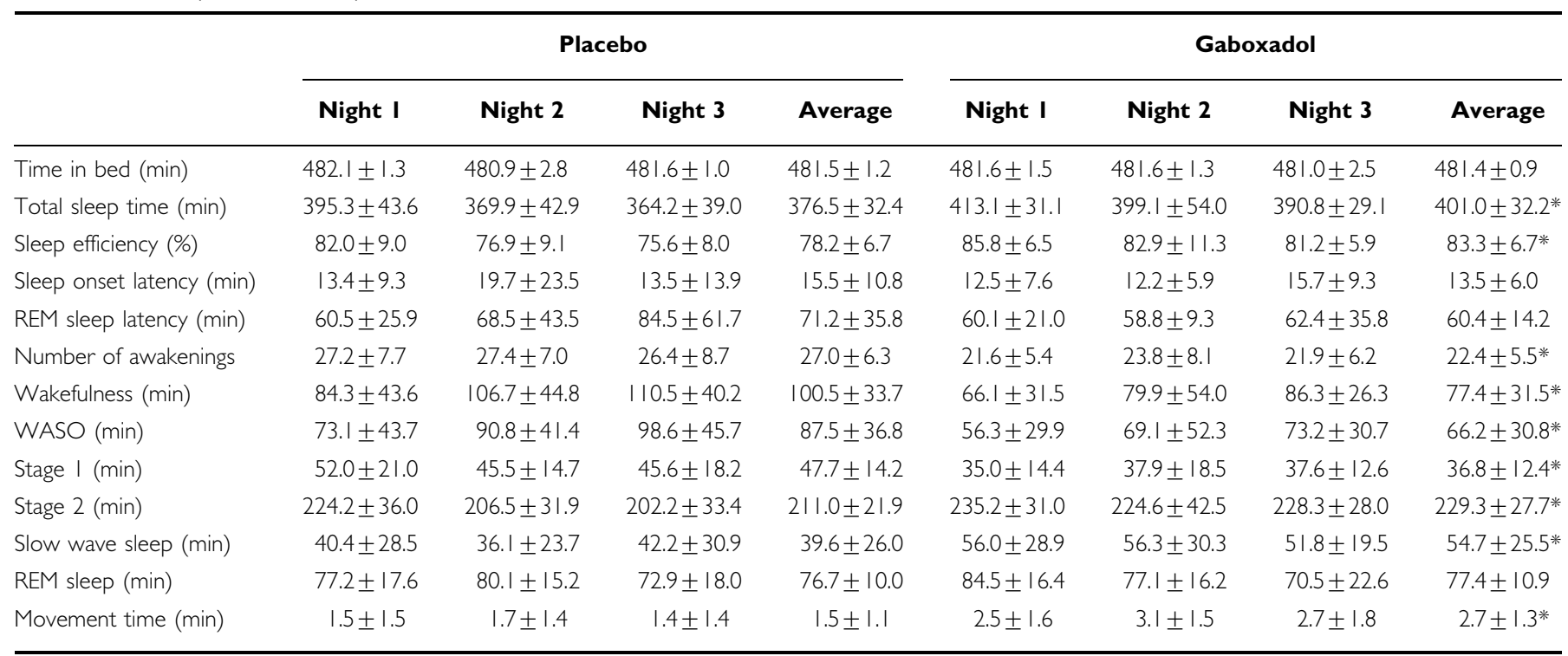

Values are means $\pm S D(n=10)$. Average, mean of three placebo or gaboxadol nights. WASO, wakefulness after sleep onset.

*Significant difference between the treatments ( $P<0.05$, ANOVAeffect of treatment). ANOVA was applied to log-transformed data.

significant effect of treatment $(\mathrm{F}(1,9)=20.6, P<0.01)$, time $(\mathrm{F}(3,27)=25.8, \quad P<0.0001)$ and of treatment by time $(\mathrm{F}(3,27)=3.5, P<0.05)$ was found, due to the fact that the gaboxadol-induced increases mainly occurred during the first half of the night.

\section{EEG Spectral Power Densities}

Analysis of SWA in the EEG within non-REM sleep found a significant effect of treatment $(\mathrm{F}(1,9)=10.4, P<0.01)$, reflecting an overall enhancement of SWA by gaboxadol (Figure 2 lower plot). Furthermore, the effect of time was significant $(F(3,27)=36.9, P<0.0001)$, which was caused by a monotonic decrease across the placebo and gaboxadol nights.

ANOVAs of the EEG power densities within non-REM sleep in each of the $0.5 \mathrm{~Hz}$ bins found a significant $(P<0.05)$ effect of treatment for the frequency bands between 0.5 and $2.5 \mathrm{~Hz}$, between 4.5 and $7 \mathrm{~Hz}$, and from 13.5 up to $14.5 \mathrm{~Hz}$. Independent of the treatment night, gaboxadol elevated EEG activity in the delta and theta frequency region and suppressed power densities in a part of the sigma frequency range throughout the night (Figure 2). A significant time effect emerged for nearly all frequency bands, caused by a general declining trend.

\section{Cognitive Performance and Mood}

None of the performance measures revealed significant differences between the placebo and gaboxadol treatment. For the target detection test, a significant effect of treatment day was found $(\mathrm{F}(2,18)=28.5, P<0.0001)$. Irrespective of the treatment, the number of correct responses increased across the treatment days (Figure 3). Neither the stroop task nor the immediate word recall test yielded significant effects. For visual form recognition, a significant effect of time was found $(\mathrm{F}(2,18)=15.4, P<0.001)$, due to a general reduction in the number of correct recognitions in the course of the day. During both placebo and gaboxadol, performance on the digit span backwards test improved over the treatment days (ANOVA, effect of 'treatment day' $\mathrm{F}(2,18)=7.4, P<0.01)$. For mood and self-rated efficiency, no significant effects emerged.

\section{DISCUSSION}

During the placebo condition, the sleep of our subjects was in good agreement with earlier reports on the quantity and quality of sleep in elderly people (reviewed in Miles and Dement, 1980; Bliwise, 1993). They typically displayed a low sleep efficiency, which was not due to abnormal sleep onset latencies, but due to a high amount of intermittent wakefulness, particularly during the second part of the night. Although considerable for their age, the amount of slow wave sleep is lower than that usually observed in young subjects (eg Dijk et al, 1989; Landolt et al, 1996; Mathias et al, 2001). The age-related reduction in slow wave sleep is reportedly associated with a decrease in the amplitude (Feinberg et al, 1967) and number (Ehlers and Kupfer, 1989) of slow waves and, consequently, with an attenuation of spectral power density in the delta frequencies (Dijk et al, 1989; Ehlers and Kupfer, 1989; Landolt et al, 1996).

No subjects reported negative side effects. Therefore, conforming with an earlier study (Lancel et al, 2001), the employed dose of $15 \mathrm{mg}$ of gaboxadol hydrochloride was well tolerated. This dose of gaboxadol exerted clear effects on subjectively assessed sleep parameters; it significantly shortened self-estimated sleep onset latency, on average by $10 \mathrm{~min}$, tended to increase the total sleep time (ANOVA, effect of treatment $F(1,9)=3.2, P<0.1)$ and to reduce the number of awakenings $(F(1,9)=4.6, P<0.1)$ and significantly increased the self-rated quality as well as intensity of sleep. Polysomnographic recordings confirmed the hypno- 

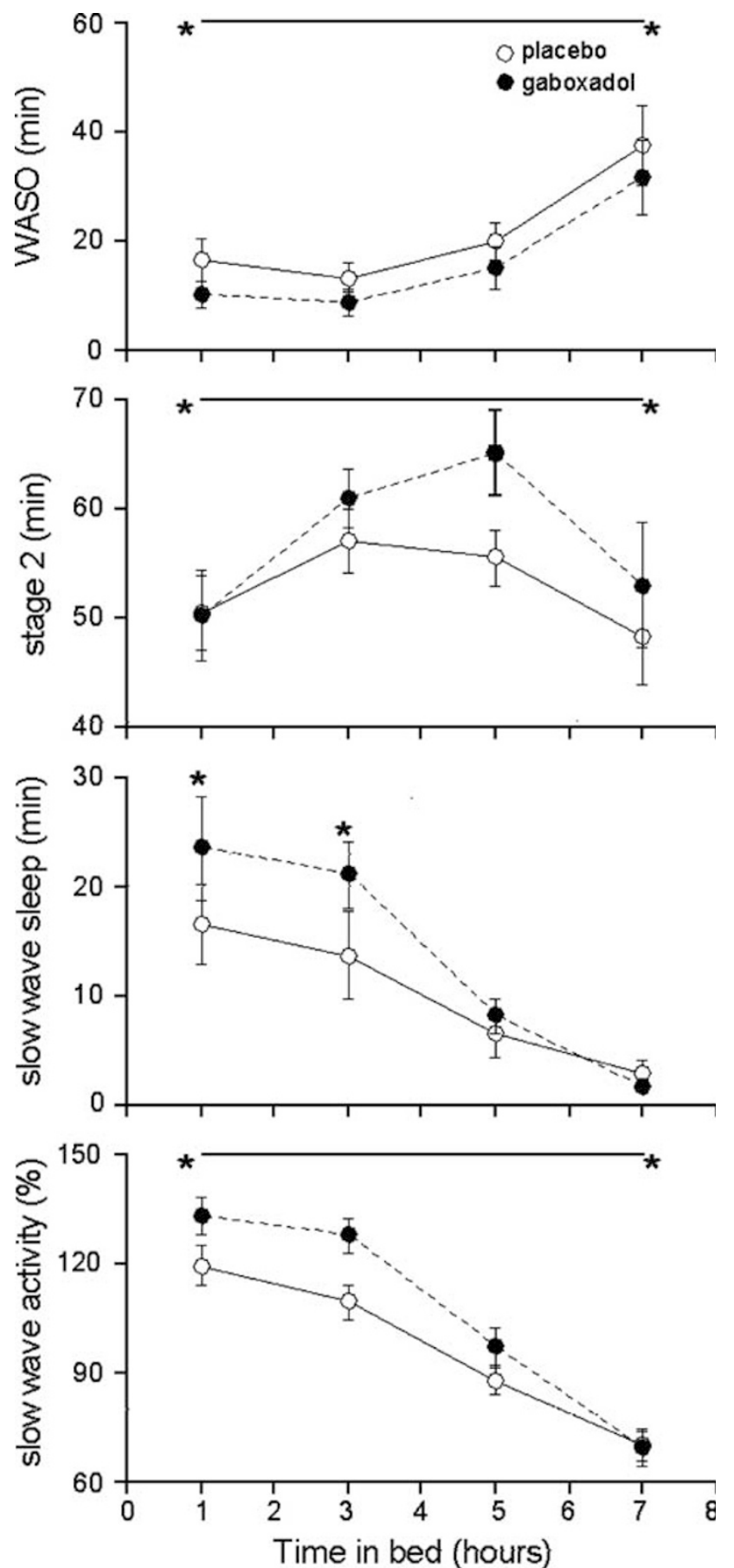

Figure I Time course of wakefulness after sleep onset (WASO), stage 2, slow wave sleep, and average SWA in the EEG within non-REM sleep during the placebo and gaboxadol nights. Values are means \pm SEM $(n=10)$ and are plotted in the middle of the 2-h intervals. Placebo, mean of three placebo nights. Gaboxadol, mean of three gaboxadol nights. For each subject, SWA data were expressed as percentage of average SWA within non-REM sleep during all placebo nights. *__ $*$ Significant treatment effect (ANOVA, $P<0.05$ ). *Significant difference between the treatments $(P<0.05$, tests with contrasts). Statistical procedures were applied to logtransformed data.

tic action of gaboxadol. Gaboxadol improved sleep consolidation, as it significantly decreased the number of awakenings and the amount of wakefulness after sleep onset. Furthermore, it augmented deep sleep, in that it

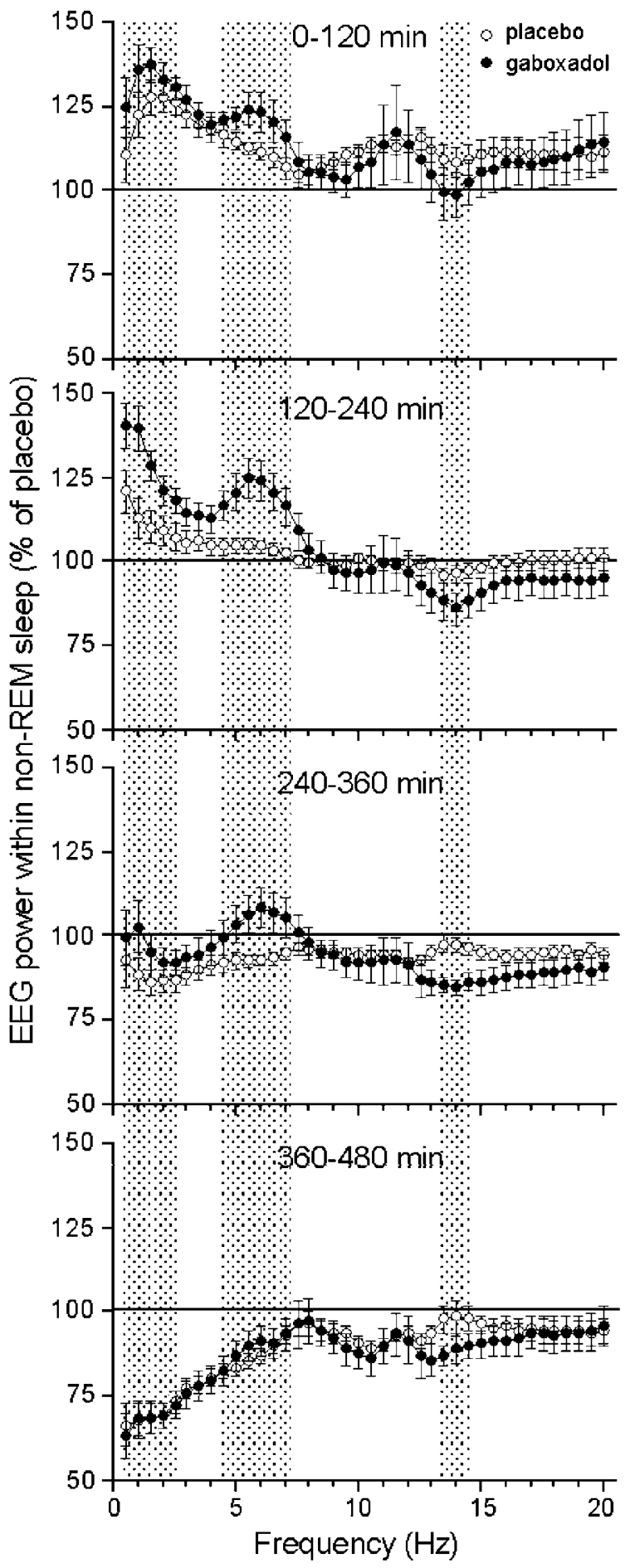

Figure 2 EEG power densities within non-REM sleep per 2-h interval during the placebo and gaboxadol nights. Values are means $\pm \operatorname{SEM}(n=10)$. Placebo, mean of three placebo nights. Gaboxadol, mean of three gaboxadol nights. For each subject, the values were expressed as percentage of the average EEG power density in the same frequency band within non-REM sleep during the entire placebo nights. Shaded areas refer to frequency bands for which a significant treatment effect was found (ANOVA, $P<0.05$ ). ANOVA was applied to log-transformed data.

reduced not only wakefulness, but also stage 1, while increasing slow wave sleep, particularly during the beginning of the night, as well as stage 2, mainly later during the night. The increase in sleep intensity is corroborated by the 

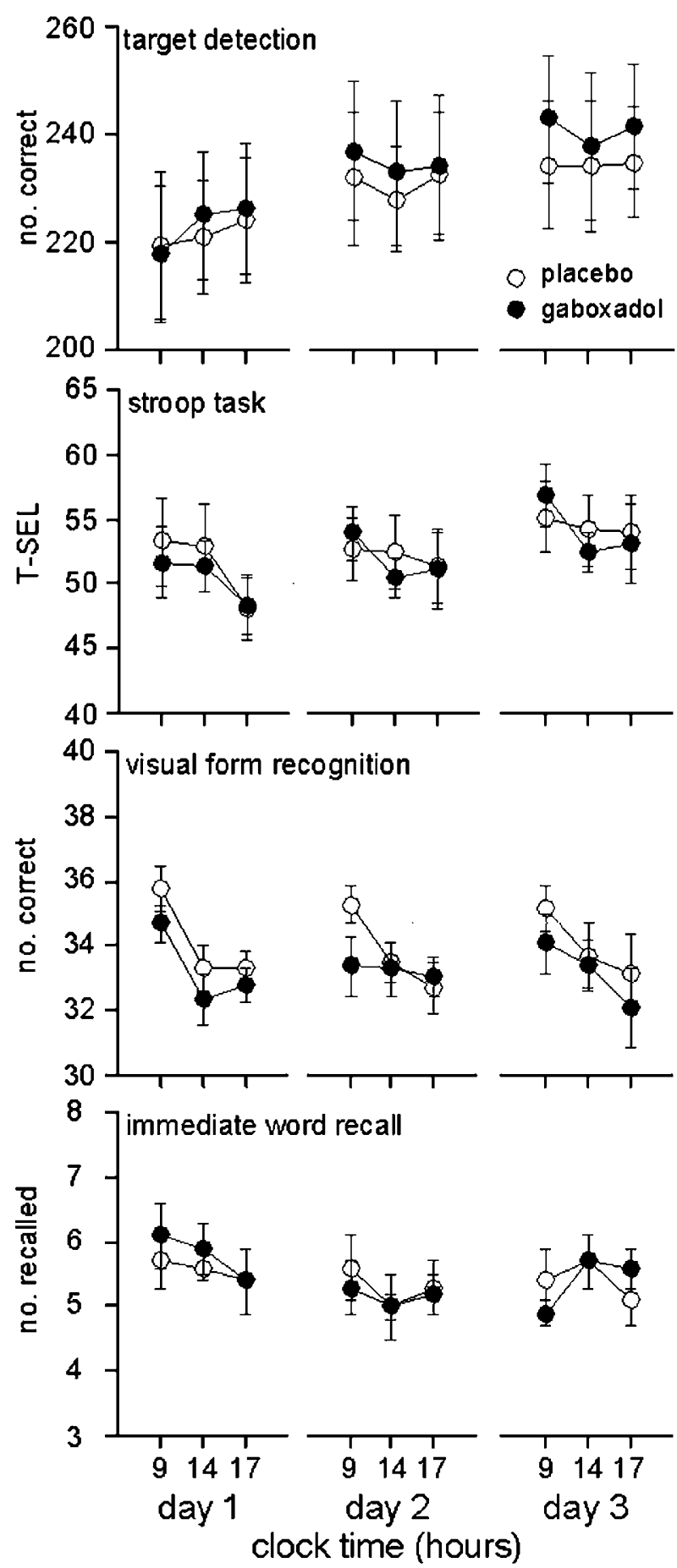
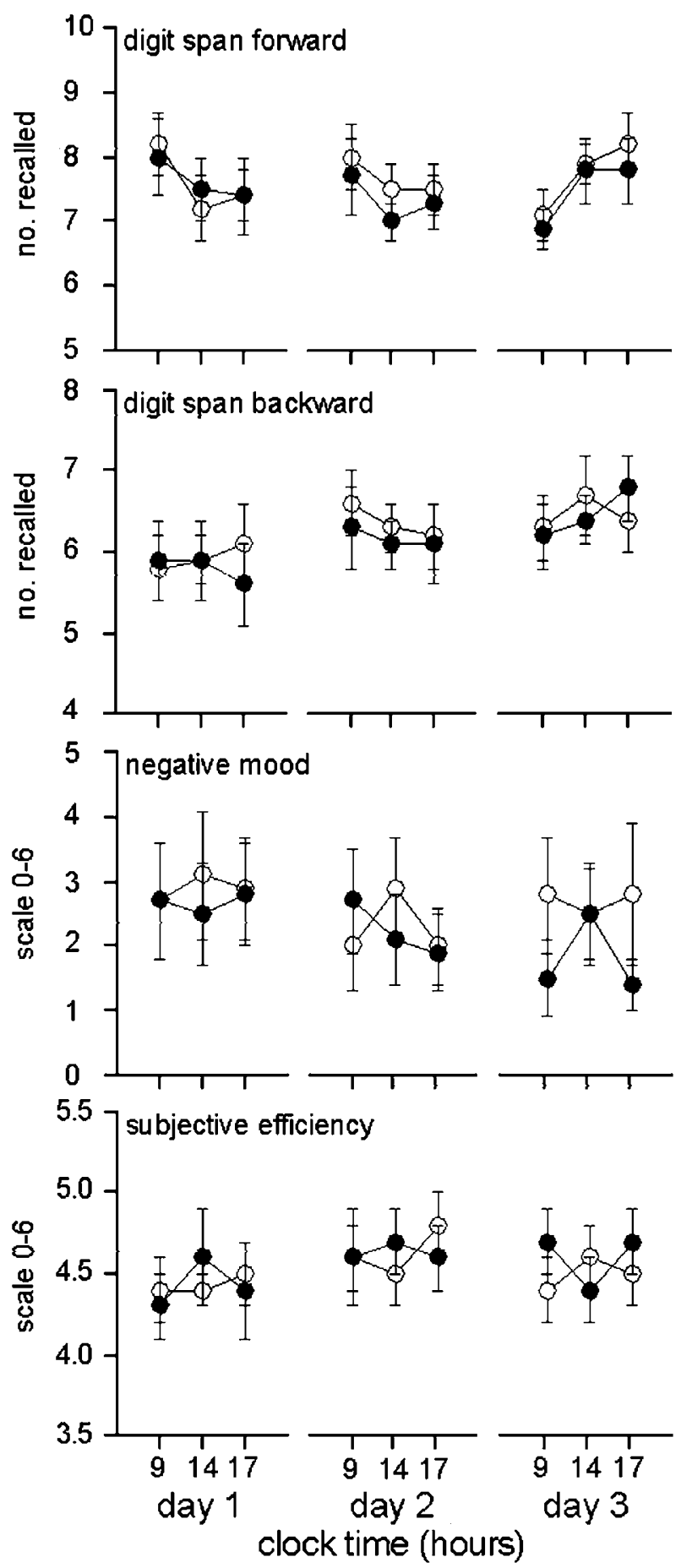

Figure 3 Cognitive performance and mood assessments during the placebo and gaboxadol days. Values are means \pm SEM ( $n=10)$.

influence of gaboxadol on the EEG signals during non-REM sleep. Spectral analysis found that gaboxadol significantly enhanced power in the delta and theta frequency bands and reduced sigma activity. These observations confirm earlier reports, as comparable subjective and objective effects were found in previous studies on the influence of a single dose of gaboxadol on normal night sleep in young and aged subjects (Faulhaber et al, 1997; Lancel et al, 2001) and/or on post-nap night sleep in young men (Mathias et al, 2001). A novel finding is that the hypnotic effects of gaboxadol in elderly subjects are sustained during subchronic treatment. In agreement with earlier observations in rats (Lancel and Langebartels, 2000), this finding suggests that gaboxadol may not rapidly produce tolerance toward its sleep effects. Moreover, subchronic gaboxadol administration revealed a rather intriguing effect on self-rated fitness upon awakening. While we never found an effect of acute gaboxadol administration on this parameter, the present study demonstrates that subjects feel slightly, but significantly, more refreshed after three gaboxadol nights than after three 
placebo nights. Possibly, the gaboxadol-induced improvement of sleep quality may, after a number of nights, express itself in feeling more rested in the morning. However, this result needs to be confirmed.

The cognitive performance and mood assessment battery failed to find negative effects of $15 \mathrm{mg}$ of gaboxadol hydrochloride on any of the attention, memory, and mood measures. This finding suggests that, when administered at this dose, gaboxadol does not impair the ability to concentrate and perform during the following day. In view of its short elimination half-life, one may not have expected residual effects. However, as documented for a number of benzodiazepines (Hindmarch and Ott, 1984) and zolpidem (Roth et al, 1995; Troy et al, 2000; Hindmarch et al, 2001), hypnotic substances with short half-lives are not necessarily free from residual effects. Moreover, as the sleep effects of gaboxadol appear longer-lasting in aged (present study; Lancel et al, 2001) than in young subjects (Faulhaber et al, 1997; Mathias et al, 2001), its elimination half-life probably increases with advancing age.

Insomniacs often complain of fatigue-related problems, including concentration problems, memory difficulties, and poor performance (reviewed in Costa e Silva et al, 1996; Walsh and Ustun, 1999). As gaboxadol improves sleep quality - as reflected particularly by increases in sleep continuity and slow wave sleep-it might be expected to increase rather than impair next-day performance. However, we found no positive influence of gaboxadol on any of the attention, memory, and mood parameters. Various explanations can be given for the absence of performanceincreasing effects. First, it is still questionable whether sleep quality has such an impact on daytime functioning. For instance, in one study, three groups of chronic insomniacs were treated during 3 weeks with placebo, $30 \mathrm{mg}$ of the benzodiazepine temazepam or $7.5 \mathrm{mg}$ zopiclone. Although both hypnotics were effective in that they persistently shortened self-reported sleep onset latency and increased self-rated sleep depth, they did not modify daytime alertness nor cognitive functioning (Stip et al, 1999). Further, studies investigating the relationship between sleep quality or the amount of slow wave and next-day performance failed to produce consistent results (for references, see Walsh and Ustun, 1999; Edinger et al, 2000). Finally, for their age group, our subjects were physically and mentally very healthy and had no complaints about their normal sleep. Probably as a consequence, they already performed so well during the placebo condition, that it may be hard to elicit and detect improvements. In future, it may be worthwhile to investigate the impact of repeated gaboxadol administration on next-day performance in insomniac patients or in subjects with experimentally disturbed sleep and/or cognitive performance.

In conclusion, the present study shows that $15 \mathrm{mg}$ gaboxadol hydrochloride exerts marked effects on night sleep in healthy elderly subjects - it significantly reduces self-reported sleep onset latency and increases self-rated sleep quality and intensity, decreases the number of awakenings and intermittent wakefulness, promotes slow wave sleep and stage 2, enhances low-frequency activity in the EEG within non-REM sleep, while hardly affecting REM sleep - that to a large extent reverse the typical age-related sleep changes. The study also demonstrates that the effects of gaboxadol on subjective and objective sleep parameters are stable throughout the three-night treatment period, indicating that tolerance to its sleep effects may not develop very rapidly. Additionally, this study reveals that $15 \mathrm{mg}$ of gaboxadol does not exert deleterious effects on attention, memory, and mood during the following days. Taken together, these findings suggest that gaboxadol, when administered in this dose, is an effective hypnotic, in that it improves sleep, without producing adverse or residual effects in elderly people. In view of the intriguing effects observed in this small pilot study, it seems worthwhile to replicate this experiment in a larger sample.

\section{ACKNOWLEDGEMENTS}

We greatly acknowledge $\mathrm{H}$ Lundbeck A/S, Copenhagen, Denmark for their financial support, $G$ Dirlich for his software support, and A Wendl for the expert administration of the test battery.

\section{REFERENCES}

Bäumler G (1985). Farbe-Wort-Interferenztest (FWIT) nach J.R. Stroop. Verlag für Psychologie: Göttingen, Toronto, Zürich.

Benton AL, Hamsher K de S, Varney NR, Spreen O (1983). Contributions to Neuropsychological Assessment. Oxford University Press: New York.

Billiard M, Besset A, de Lustrac C, Brissaud L (1987). Doseresponse effects of zopiclone on night sleep and on nighttime and daytime functioning. Sleep 10(Suppl 1): 27-34.

Bliwise DL (1993). Sleep in normal aging and dementia. Sleep 16: 40-81.

Brickenkamp R (1995). Aufmerksamkeitsbelastungstest 'd2', erweiterte und neu gestaltete Auflage. Diagnostica 41: 291-296.

Broadhurst A, Cushnaghan RC (1987). Residual effects of zopiclone (imovane). Sleep 10(Suppl 1): 48-53.

Costa e Silva JA, Chase M, Sartorius N, Roth T (1996). Special report from a symposium held by the World Health Organization and the World Federation of Sleep Research Societies: an overview of insomnias and related disorders-recognition, epidemiology, and rational management. Sleep 19: 412-416.

Dijk DJ, Beersma DGM, van den Hoofdakker RH (1989). All night spectral analysis of EEG sleep in young and middle-aged male subjects. Neurobiol Aging 10: 677-682.

Edinger JD, Glenn DM, Bastian LA, Marsh GR (2000). Slow-wave sleep and waking cognitive performance II: findings among middle-aged adults with and without insomnia complaints. Physiol Behav 70: 127-134.

Ehlers CL, Kupfer DJ (1989). Effects of age on delta and REM sleep parameters. Electroencephalogr Clin Neurophysiol 72: 118-125.

Faulhaber J, Steiger A, Lancel M (1997). The GABA Agonist THIP produces slow wave sleep and reduces spindling activity in NREM sleep in humans. Psychopharmacology 130: 285-291.

Feinberg I, Koresko RL, Heller N (1967). EEG sleep patterns as a function of normal and pathological aging in man. J Psychiatr Res 5: 107-144.

Gorenstein C, Gentil V (1983). Residual and acute effects of flurazepam and triazolam in normal subjects. Psychopharmacology 80: 376-379.

Greenblatt DG, Allen MD (1978). Toxicity of nitrazepam in the elderly: a report from the Boston Collaborating Drug Surveillance Program. Br J Clin Pharmacol 5: 407-413.

Greenblatt DG, Allen MD, Shader RI (1977). Toxicity of high dose flurazepam in the elderly. Clin Pharmacol Ther 21: 355-361. 
Harrison C, Subhan Z, Hindmarch I (1985). Residual effects of zopiclone and benzodiazepine hypnotics on psychomotor performance related car driving. Drugs Exp Clin Res XI: 823-829.

Hedden T, Gabrieli JDE (2004). Insight into the ageing mind: a view from cognitive neuroscience. Nat Neurosci 5: 87-97.

Hemmeter U, Müller M, Bischof R, Annen B, Holsboer-Trachsler E (2000). Effect of zopiclone and temazepam on sleep EEG parameters, psychomotor and memory functions in healthy elderly subjects. Psychopharmacology 147: 384-396.

Hindmarch I, Fairweather DB (1994). Assessing the residual effects of hypnotics. Acta Psychiat Belg 94: 88-95.

Hindmarch I, Ott H (1984). Sleep, benzodiazepines and performance: issues and comments. In: Hindmarch I, Ott $\mathrm{H}$, Roth $\mathrm{T}$ (eds). Psychopharmacology Supplement 1. Springer-Verlag: Berlin. pp 194-202.

Hindmarch I, Patat A, Stanley N, Paty I, Rigney U (2001). Residual effects of zaleplon and zolpidem following middle of the night administration five hours to one hour before awakening. Hum Psychopharmacol Clin Exp 16: 159-167.

Janke W, Erdmann G, Hüppe M, Debus G (1999). Befindlichkeitsskalierung anhand von Kategorien und Eigenschaftswörtern (BSKE). Unpublished Manual. Institut für Psychologie der Universität: Würzburg.

Kunz D, Herrmann WM (2000). Sleep-wake cycle, sleep-related disturbances, and sleep disorders: a chronobiological approach. Comprehens Psychiatry 41: 104-115.

Lancel M (1997). The GABA $_{A}$ agonist THIP increases non-REM sleep and enhances non-REM sleep-specific delta activity in the rat during the dark period. Sleep 20: 1099-1104.

Lancel M (1999). Role of $\mathrm{GABA}_{\mathrm{A}}$ receptors in the regulation of sleep: initial sleep responses to peripherally administered modulators and agonists. Sleep 22: 33-42.

Lancel M, Langebartels A (2000). Aminobutyric $\operatorname{acid}_{\mathrm{A}}\left(\mathrm{GABA}_{\mathrm{A}}\right)$ agonist 4,5,6,7-tetrahydroisoxazolo(4,5-c)pyridin-3-ol persistently increases sleep maintenance and intensity during chronic administration to rats. J Pharmacol Exp Ther 293: 1084-1090.

Lancel M, Faulhaber J (1996). The $\mathrm{GABA}_{\mathrm{A}}$ agonist THIP (gaboxadol) increases non-REM sleep and enhances delta activity in the rat. Neuroreport 7: 2241-2245.

Lancel M, Wetter TC, Steiger A, Mathias M (2001). Effect of the $\mathrm{GABA}_{\mathrm{A}}$ agonist gaboxadol on nocturnal sleep and hormone secretion in healthy elderly subjects. Am J Physiol 281: E130-E137.

Landolt HP, Dijk DJ, Achermann P, Borbély AA (1996). Effect of age on the sleep EEG: slow-wave activity and spindle frequency activity in young and middle-aged men. Brain Res 738: 205-212.

Lezak M (1995). Neuropsychological Assessment, 3rd edn. Oxford University Press: New York, Oxford.

Mathias S, Steiger A, Lancel $M$ (2001). The $\mathrm{GABA}_{\mathrm{A}}$ agonist gaboxadol improves the quality of post-nap sleep. Psychopharmacology 157: 299-304.
Miles LE, Dement WC (1980). Sleep and aging. Sleep 3: 119-220.

Monane M (1992). Insomnia in the elderly. J Clin Psychiatry 53(Suppl 6): 23-28.

Mooris JC, Heyman A, Mohs RC, Hughes JP, van Belle G, Fillenbaum $\mathrm{G}$ et al (1989). The Consortium to Establish a Registry for Alzheimer's Disease (CERAD). Part I. Clinical and neuropsychological assessment of Alzheimer's disease. Neuro$\log y$ 39: 1159-1165.

Ohyon MM, Vecchierini MF (2002). Daytime sleepiness and cognitive impairment in the elderly population. Arch Int Med 162: 201-208.

Parrino L, Terzano MG (1996). Polysomnographic effects of hypnotic drugs. A review. Psychopharmacology 126: 1-16.

Partinen M (1993). Sleep problems in the elderly: epidemiology overview. In: Albarede JL, Morley JE, Roth T, Vellas BJ (eds). Sleep Disorders and Insomnia in the Elderly, Vol 7, Facts and Research in Gerontology. Serdi: Paris. pp 11-16.

Rechtschaffen A, Kales A (1968). A Manual of Standardized Terminology, Techniques and Scoring System for Sleep Stages of Human Subjects. National Institute of Health Publications, US Government Printing Office: Washington, DC.

Roehrs T, Kribbs N, Zorick F, Roth T (1986). Hypnotic residual effects of benzodiazepines with repeated administration. Sleep 9: 309-316.

Roth T, Roehrs T, Vogel G (1995). Zolpidem in the treatment of transient insomnia: a double-blind, randomized comparison with placebo. Sleep 18: 246-251.

Scharf MB, Mayleben DW, Kaffeman M, Krall R, Ochs R (1991). Dose response effects of zolpidem in normal geriatric subjects. J Clin Psychiatry 52: 77-83.

Schultz B, Aaes-Jørgensen T, B $\varnothing$ ges $\varnothing$ KP, Jørgensen A (1981). Preliminary studies on the absorption, distribution, metabolism, and excretion of THIP in animal and man using ${ }^{14} \mathrm{C}$-labelled compound. Acta Pharmacol Toxicol 49: 116-124.

Seppala M, Rajala T, Sourander L (1993). Subjective evaluation of sleep and the use of hypnotics in nursing homes. Aging 5: 199-205.

Stip E, Furlan M, Lussier I, Bourgouin P, Elie R (1999). Doubleblind, placebo-controlled study comparing effects of zopiclone and temazepam on cognitive functioning of insomniacs. Hum Psychopharmacol Clin Exp 14: 253-261.

Troy SM, Lucki I, Unruh MA, Cevallos WH, Leister CA, Martin PT et al (2000). Comparison of the effects of zaleplon, zolpidem, and triazolam on memory, learning, and psychomotor performance. J Clin Psychopharmcol 20: 328-337.

Tuokko H, Frerichs RJ (2000). Cognitive impairment with no dementia (CIND): longitudinal studies, the findings, and the issues. Clin Neuropsychologist 14: 504-525.

Walsh J, Ustun TB (1999). Prevalence and health consequences of insomnia. Sleep 22(Suppl 3): S427-S436. 